\title{
Rational modelling of elastic soil behaviour in 3D condition
}

\author{
Teruo Nakai ${ }^{1, *}$, Hossain Md. Shahin ${ }^{2}$, and Akira Ishikawa $^{3}$ \\ ${ }^{1}$ Nagoya office, Geo-Research Institute, Nagoya, Japan \\ ${ }^{2}$ Department of Civil and Environment Engineering, Islamic University of Technology, Dhaka, Bangladesh \\ ${ }^{3}$ Technical Research Centre, Shimizu Corporation, Tokyo, Japan
}

\begin{abstract}
A simple and rigorous formulation of elastic component of elastoplastic model for geomaterials is presented. Although linear relation between elastic volumetric strain and mean principal stress in log scale is assumed in most of the usual models, linear relation between each principal stress and the corresponding principal elastic strain in log scale is assumed. Incorporating Poisson's ratio, three principal stresses vs. three elastic principal strain relation is obtained. Also, assuming coaxially between stresses and elastic strains, this relation can be transformed to stress- elastic strain relation in general coordinate. The material parameters of the proposed model of the elastic component are the same as those of the usual models, i.e., swelling index $\kappa$ and Poisson's ratio $v$. This proposed model can describe typical unloading behaviour of various shear tests and constant stress ratio unloading tests reported before.
\end{abstract}

\section{Introduction}

In 3D elastoplastic modelling, though there have been many discussions on the modelling of plastic component, few attentions are paid to elastic component. Elastic component of most elastoplastic models is expressed by incremental non-linear Hooke's law using the swelling index $\kappa$ and the Poisson's ratio $v$. However, according to this modelling, the elastic volumetric stain is independent of the stress path, but other strains (e.g., deviatoric strain) depend on the stress path. Particularly, implicit formulation such as return mapping in elastoplastic analysis requires unique relation not between stress increments and elastic strain increments but between stresses and elastic strains. Also, there is a possibility for the stress condition to enter into tension zone in the formulation using Hooke's law. Generation of tensile stress during elastic deformation causes instability of calculation when adopting the elastoplastic constitutive model to boundary value problems.

\section{Ordinary modelling of elastic component and its problem}

In most ordinary elastoplastic models of geomaterials, the elastic component is formulated using the incremental Hooke's law. The principal strain increments are expressed using the principal stress increments as follows:

$$
\left\{\begin{array}{l}
d \varepsilon^{(1)} \\
d \varepsilon^{(2)} \\
d \varepsilon^{(3)}
\end{array}\right\}=\frac{1}{E}\left[\begin{array}{ccc}
1 & -v & -v \\
-v & 1 & -v \\
-v & -v & 1
\end{array}\right]\left\{\begin{array}{l}
d \sigma^{(1)} \\
d \sigma^{(2)} \\
d \sigma^{(3)}
\end{array}\right\}
$$

General Hooke's law in incremental form is given by:

\footnotetext{
* Corresponding author: nakai.teruo@nitech.ac.jp
}

$$
d \sigma_{i j}=\frac{E}{1+v} d \varepsilon_{i j}+\frac{v E}{(1+v)(1-2 v)} d \varepsilon_{k k} \delta_{i j}
$$

Here, tangential Young's modulus $E$ is proportional to mean principal stress $p$ and expressed as follows using the swelling index $\kappa$ on $e-\ln p$ relation in isotropic or $K \mathrm{O}$ unloading compression tests, and Poisson's ratio $v$ :

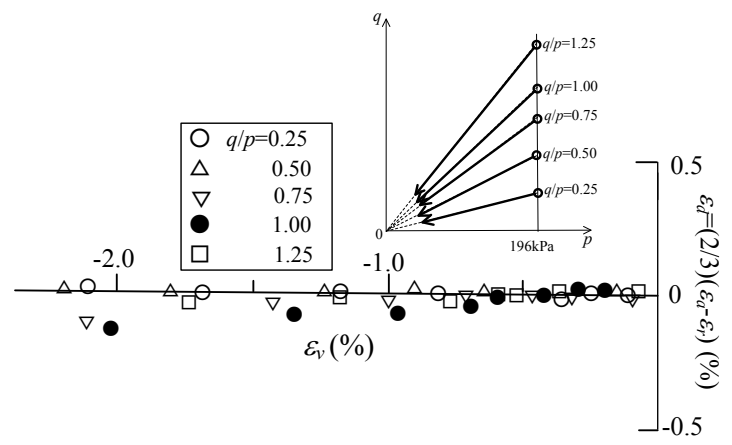

Fig. 1. Observed results of clay under constant stress ratio unloading conditions (Ohomaki [1])

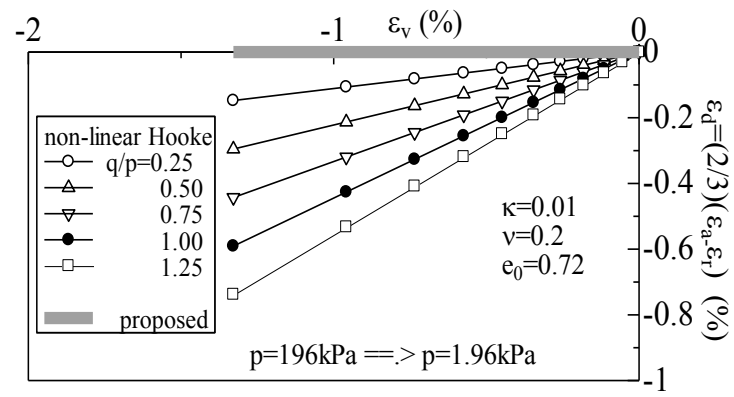

Fig. 2. Calculated results of clay under constant stress ratio unloading conditions 


$$
E=\frac{3(1-2 v)\left(1+e_{0}\right)}{\kappa} p \quad\left(e_{0}: \text { initial void ratio }\right)
$$

Figure 1 shows the observed relation between volumetric strain $\varepsilon_{v}$ and deviatoric strain $\varepsilon_{d}$ of a normally consolidated clay under constant stress ratio $(q / p=$ const.) unloading tests [1]. It can be seen from this figure that the elastic strain is always isotropic regardless of the stress ratio, because elastic strain alone occurs in these stress paths. Same tendency on clay and sand was reported in the references [2, 3]. The lines of Fig. 2 show the corresponding results calculated by Eq. (1), arranged with the same relation as Fig. 1, where material parameters $\kappa=0.01, v=0.2, e_{0}=0.72$. In every case mean stress is decreased from $196 \mathrm{kPa}$ to $1.96 \mathrm{kPa}$. Although the volumetric strain is independent of the stress ratio, deviatoric strain becomes large with increase of stress ratio.

Rectangular dots in Fig. 3 show the results calculated by Eq. (1) in terms of the relation between $\varepsilon_{v}$ and $\varepsilon_{d}$, when stress changes from point $\mathrm{O}$ to point $\mathrm{A}$ under different stress paths (O-B-A and O-C-A). In these stress paths, only elastic strain occurs. It can be recognized that the elastic deviatoric strain calculated by Eq. (1) depends on the stress path, even if the initial and targeted stresses are same. This means that the strain obtained by Eqs. (1) to (3) is not fully elastic in true sense.

Figure 4 illustrates a schematic yield surface of Cam clay type's model in $p-q$ plane. In this figure, the upper half is in triaxial compression condition, and the lower half is in triaxial extension condition. The broken lines $q / p=3.0$ and $q / p=1.5$ indicate the stress condition where minor principal stress is zero. Here, the area without tensile stress is limited in the zone of green colour. As shown in this figure, the yield surface $(f=0)$ of Cam clay type model, which is formulated by the stress parameters ( $p$ and $q$ ) and is symmetric with respect to $p$-axis, has tension zone inside of the yield surface. This means that tension stress may develop during elastic deformation. Also, even in elastoplastic region (stress condition lies on the yield surface), tensile stress occurs for stress ratio $q / p>3.0$ in triaxial compression and $q / p>1.5$ in triaxial extension. Further, when implicit formulation such as return mapping algorithm is adopted, first trial stress is obtained assuming elastic state. In this trial step, the stress condition may enter the tension zone if Hooke's elasticity is used.

As is understood from the above discussion, a formulation of elastic component which never develops tension stress under any kinds of deformation is desired. This is because geomaterials are governed by frictional law and have no resistance against tensile stress.

\section{Elastic model without generating tensile stress}

\subsection{Formulation of model}

Considering the test results of isotropic deformation (Fig. 1) and well-known linear relation between void ratio and
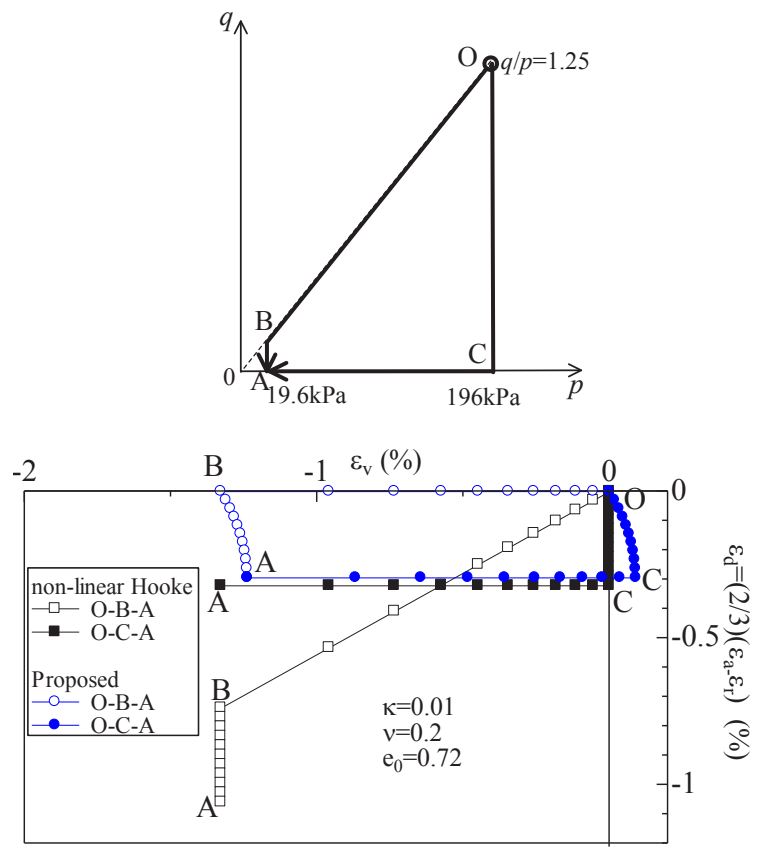

Fig. 3. Calculated results of clay under unloading stress paths O-B-A and O-C-A

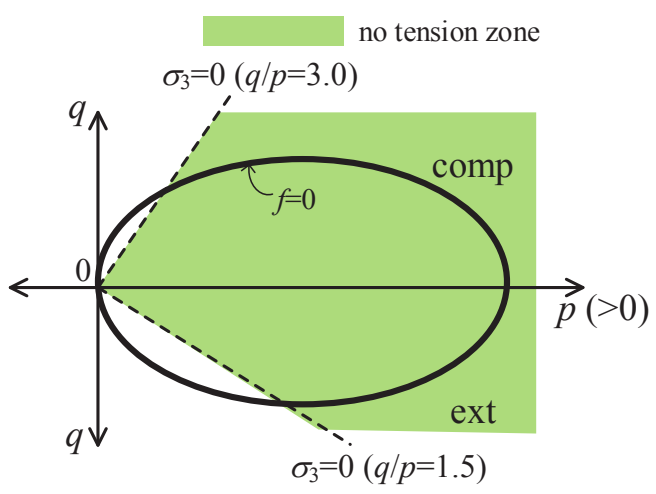

Fig. 4._Zone where tension stress never occurs in $(p, q)$ plane and yield surface of ordinary elastoplastic model

stress in log scale under constant stress ratio unloading condition, three principal elastic strains are given by the following equation [4]:

$$
\left\{\begin{array}{l}
\varepsilon^{(1)} \\
\varepsilon^{(2)} \\
\varepsilon^{(3)}
\end{array}\right\}=\frac{1}{S}\left[\begin{array}{ccc}
1 & -v & -v \\
-v & 1 & -v \\
-v & -v & 1
\end{array}\right]\left\{\begin{array}{l}
\ln \left(\sigma^{(1)} / p_{a}\right) \\
\ln \left(\sigma^{(2)} / p_{a}\right) \\
\ln \left(\sigma^{(3)} / p_{a}\right)
\end{array}\right\}
$$

where, $P_{a}$ is atmospheric pressure. This equation is obtained only by replacing three principal stresses in Hooke's law by their logarithm. From Eq. (4), three principal stresses are expressed as the functions of three principal elastic strains. 


$$
\begin{aligned}
\sigma^{(1)}= & P_{a} \exp \left\{\Delta\left(\varepsilon^{(1)}+\frac{v}{1-v} \varepsilon^{(2)}+\frac{v}{1-v} \varepsilon^{(3)}\right)\right\}, \\
\sigma^{(2)}= & P_{a} \exp \left\{\Delta\left(\frac{v}{1-v} \varepsilon^{(1)}+\varepsilon^{(2)}+\frac{v}{1-v} \varepsilon^{(3)}\right)\right\}, \\
\sigma^{(3)}= & P_{a} \exp \left\{\Delta\left(\frac{v}{1-v} \varepsilon^{(1)}+\frac{v}{1-v} \varepsilon^{(2)}+\varepsilon^{(3)}\right)\right\} \\
& \text { where, } \Delta=\frac{S(1-v)}{(1+v)(1-2 v)}
\end{aligned}
$$

As can be seen from Eq. (5), three principal stresses never become negative. It can be seen from Eq. (4) that volumetric strain is expressed as:

$$
\begin{aligned}
\varepsilon_{v} & =\varepsilon^{(1)}+\varepsilon^{(2)}+\varepsilon^{(3)} \\
& =\frac{1}{S}(1-2 v)\left\{\ln \left(\sigma^{(1)} / P_{a}\right)+\ln \left(\sigma^{(2)} / P_{a}\right)+\ln \left(\sigma^{(3)} / P_{a}\right)\right\} \\
& =\frac{1}{S}(1-2 v) \ln \frac{\sigma^{(1)} \sigma^{(2)} \sigma^{(3)}}{P_{a}^{3}}=\frac{1}{S}(1-2 v) \ln \frac{I_{3}}{P_{a}^{3}}
\end{aligned}
$$

When stress changes from $\left(\sigma^{(1)}, \sigma^{(2)}, \sigma^{(3)}\right)$ to $k\left(\sigma^{(1)}\right.$, $\left.\sigma^{(2)}, \sigma^{(3)}\right)$, volumetric strain is given by:

$$
\begin{aligned}
\varepsilon_{v}= & \frac{1}{S}(1-2 v)\left\{\ln \left(k \sigma_{0}^{(1)} / \sigma_{0}^{(1)}\right)+\ln \left(k \sigma_{0}^{(2)} / \sigma_{0}^{(2)}\right)\right. \\
& \left.+\ln \left(k \sigma_{0}^{(3)} / \sigma_{0}^{(3)}\right)\right\}=\frac{3}{S}(1-2 v) \ln k
\end{aligned}
$$

and three principal strains become the same

$$
\varepsilon^{(1)}=\varepsilon^{(2)}=\varepsilon^{(3)}=\frac{1}{S}(1-2 v) \ln k
$$

Since $3(1-2 v) / S$ corresponds to $\kappa /\left(1+e_{0}\right), S$ is a constant value and is given by:

$$
S=\frac{3(1-2 v)\left(1+e_{0}\right)}{\kappa}
$$

Also, deviatoric strain $\varepsilon^{(\alpha)}-\varepsilon^{(\beta)}(\alpha \neq \beta)$ is expressed as :

$$
\begin{aligned}
\varepsilon^{(\alpha)}-\varepsilon^{(\beta)} & =\frac{1}{S}\left(\ln \frac{\sigma^{(\alpha)}}{P_{a}}-v \ln \frac{\sigma^{(\beta)}}{P_{a}}+v \ln \frac{\sigma^{(\alpha)}}{P_{a}}-\ln \frac{\sigma^{(\beta)}}{P_{a}}\right) \\
& =\frac{1}{S}(1+v) \ln \frac{\sigma^{(\alpha)}}{\sigma^{(\beta)}}
\end{aligned}
$$

Then, deviatoric strain is related not with deviatoric stress but with stress ratio.

When eigen vectors corresponding to eigen values (principal stresses; $\left.\sigma^{(\alpha)}(\alpha=1,2,3)\right)$ of stress $\sigma_{i j}$ is given by $n^{(\alpha)}{ }_{i}(i=1,2,3)$, stress $\sigma_{i j}$ can be expressed using the principal stresses and the eigen values. Also, assuming the coaxiality between stress and elastic strain, elastic principal strain $\varepsilon^{(\alpha)}$ is expressed by elastic strain $\varepsilon_{i j}$, using the eigen vectors of stress.

$$
\sigma_{i j}=\sum_{\alpha=1}^{3} \sigma^{(\alpha)} n_{i}^{(\alpha)} n_{j}^{(\alpha)}
$$
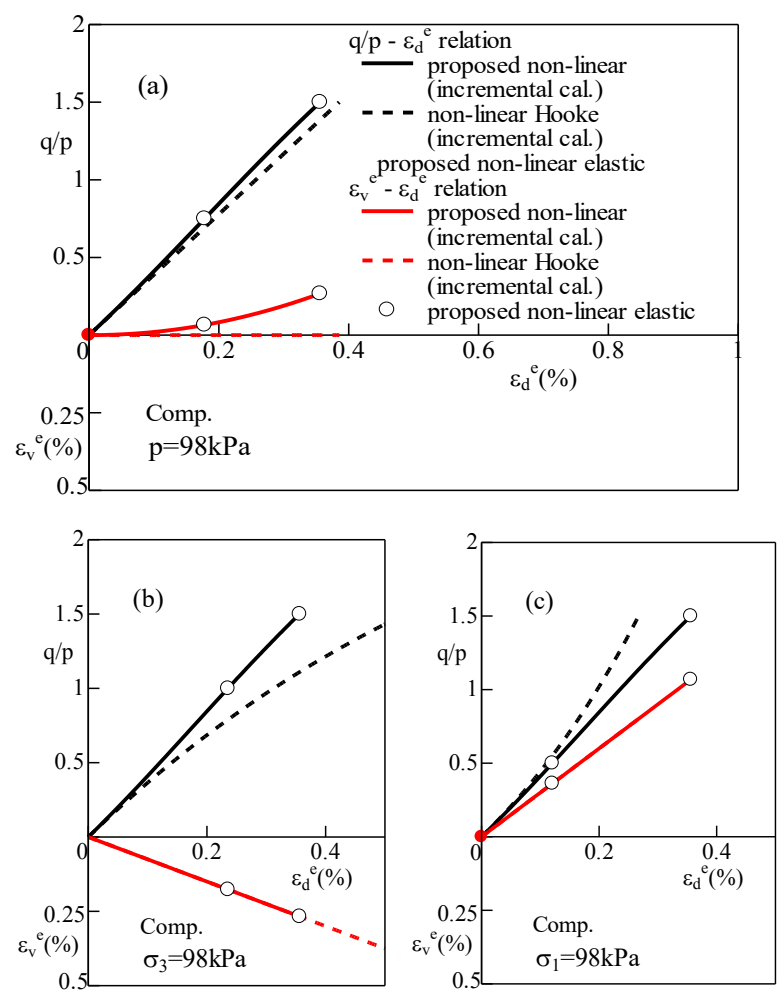

Fig. 5. Calculated $q / p-\varepsilon d^{e}-\varepsilon_{d}^{e}$ relations under $p=$ const., $\sigma_{3}=$ const. and $\sigma_{1}=$ const. condition.

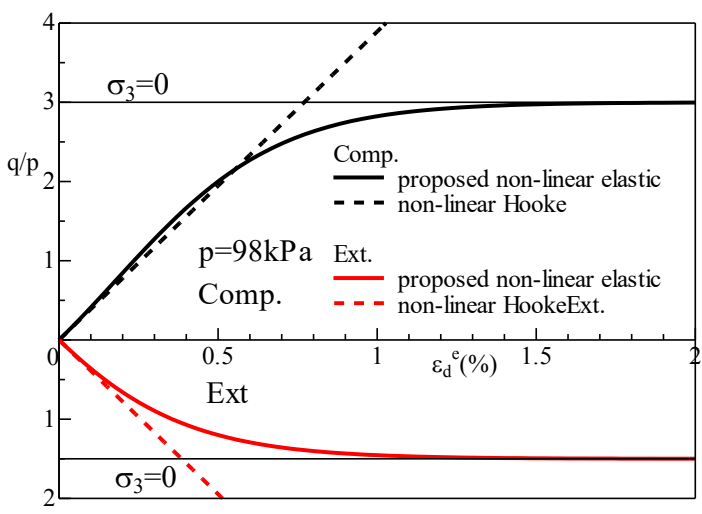

Fig. 6. Calculated $q / p-\varepsilon_{d}^{e}$ relation in triaxial compression and extension conditions.

$$
\varepsilon^{(\alpha)}=\varepsilon_{k l} n_{k}^{(\alpha)} n_{l}^{(\alpha)} \quad(\alpha=1,2,3)
$$

Using Eqs. (5), (11) and (12), $\sigma_{i j}$ can be obtained from $\varepsilon_{k l}$. Refering to the paper [5], it is possible to transform the model to the rate type model assuming the condition of coaxiality between stress and elastic strain (see Appendix).

\subsection{Simulation of elastic behaviour by proposed model}

The thick gray line in Fig. 2 is the results calculated by the proposed model. The calculated volumetric strain is the same as those of the ordinary model, but the calculated 
deviatoric strain is zero, regardless of stress ratio. The circular dots in Fig. 3 are the results by the proposed model. Since the proposed model is basically formulated as the relation between stresses and elastic strains, the strain at point $\mathrm{A}$ is independent of stress paths.

Figure 5 compares the calculated elastic strain of $p=$ const., $\sigma_{3}=$ const. and $\sigma_{1}=$ const. tests under triaxial compression condition, arranged with respect to the relation between $q / p, \varepsilon_{d}$ and $\varepsilon_{v}$. In this figure, the solid curves and the open dots represent the results by proposed model, and the dotted curves represent the results by nonlinear Hooke's law. It can be seen from the results by proposed model that elastic volumetric strain is expansive under constant mean stress, and elastic deviatoric strain is determined uniquely only by the stress ratio. Here, the open dots represent the results by Eq. (4), and the solid curves are the calculated results based on the rate type formulation. Figure 6 shows the calculated $q / p-\varepsilon_{d}$ relation by proposed model (solid curves) and non-linear Hooke's law (broken curves) in constant mean stress tests. Here, the upper half is in triaxial compression condition, and the lower half is in triaxial extension condition. Although the results by non-linear Hooke's law enter the tension zone, the results by the proposed model converge to the $\sigma_{3}=0$ lines and does not enter the tension zone with the development of the strain.

Figure 7 shows the variation of stresses $\left(\sigma_{x}, \sigma_{y}, \sigma_{z}, \tau_{x y}\right)$ by the proposed model when the strain path including rotation of principal axis is incorporated. Figure 8 shows the relation between $q / p$ and various strain components $\left(\varepsilon_{x}^{e}, \varepsilon_{y}^{e}, \varepsilon_{z}^{e}, \gamma_{x y}{ }^{e}\right)$ when the stress path including the rotation of principal stress axis is incorporated. In these figures, dots are the results calculated by Eqs. (5), (11) and (12) and curves are results from rate type formulation. It can be seen that the results derived by integration of rate type formulation agrees well with the results by Eqs. (5), (11) and (12), even when the rotation of principal axis is included in the stress path or strain path.

\subsection{Constitutive model which never generates tensile stress under any strain path}

A constitutive model which is named Suloading $t_{i j}$ model has been proposed $[6,7,8]$. This model can describe soil behaviour in general 3D condition uniquely using the concept of $t_{i j}$ [9]. Also, employing the subloading concept by Hashiguchi [10] and extending it, the influence of density, influence of bonding, time- effect and others are taken into consideration in the model.

According to the $t_{i j}$ concept, the yield surface is formulated using the stress parameters $\left(t_{N}\right.$ and $\left.t_{S}\right)$ instead of $(p$ and $q)$. Figure 9 shows the yield surface $(f=0)$ of the model based on $t_{i j}$ concept, describing on $\left(t_{N}, t_{S}\right)$ plane schematically. The upper half is in triaxial compression condition, and the lower half is in triaxial extension condition, in the same way as Fig. 4. Here, since the vertical axis ( $t_{S}$ axis) satisfies the condition of $\sigma_{3}=0$, there is no tensile stress in the region of green colour $\left(t_{N}>0\right)$. Therefore, inside and on the yield surface, all normal stresses are always positive. The elastoplastic model in which the plastic component is calculated based on $t_{i j}$

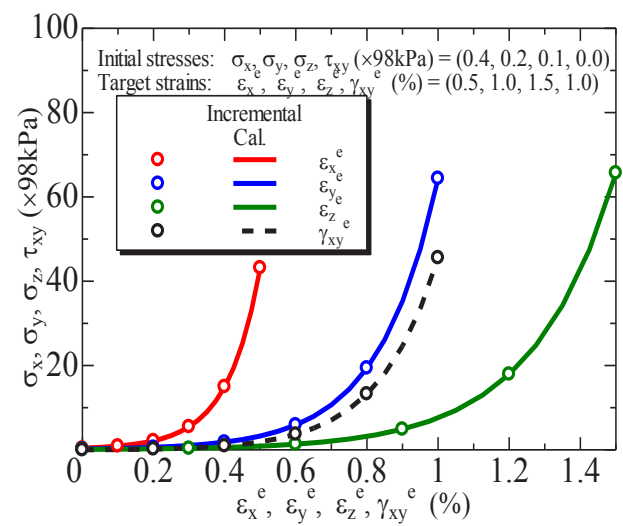

Fig. 7. Calculated stress-elastic strain relation under strain path including the rotation of principal axis

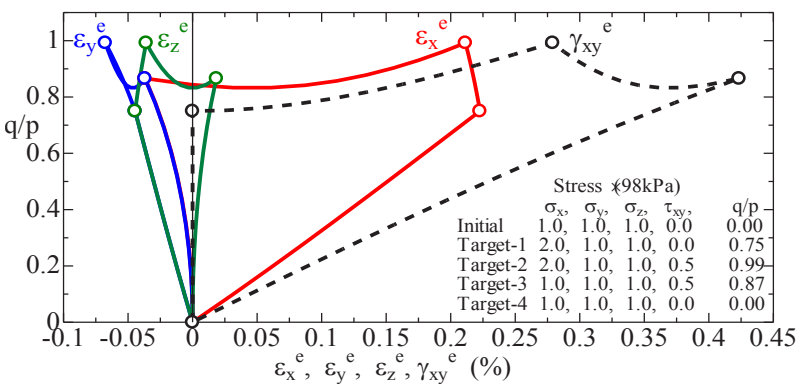

Fig. 8. Calculated stress-elastic strain relation under strain path including principal axis rotation

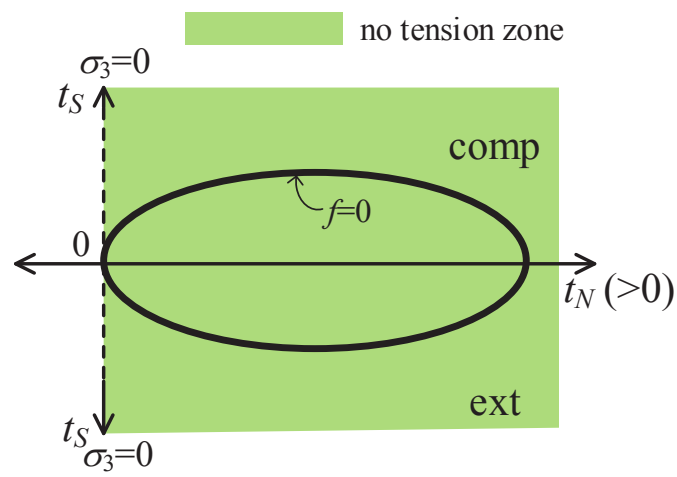

Fig.9. Zone where tensile stress never occurs in $\left(t_{N}, t_{S}\right)$ plane and yield surface of elastoplastic model based on $t_{i j}$ concept

concept and the elastic component is calculated by the present elastic model never generates tensile stress in elastoplastic deformation and elastic deformation. This is very beneficial when applying the constitutive model to the boundary value problems.

\section{Conclusions}

After describing the outline and problems of ordinary elastic model of geomaterials using incremental nonlinear Hooke's law, a new elastic model in 3D condition is presented. This model is derived by assuming a linear relation between each principal strain and the corresponding principal stress in log scale, and introducing idea of Poisson's ratio. The proposed model 
is very simple, and its material parameters are the same as those of ordinary models (swelling index $\kappa$ and Poisson's ratio $v$ ). The elastic model never generates tension stress under any strain path and simulates typical observed elastic behaviour such that linear $e-\ln p$ relation with the slope of $\kappa$ and isotropic deformation under stress ratio constant unloading test. Combining this elastic model with the elastoplastic model based on $t_{i j}$ concept (Subloading $t_{i j}$ model), it becomes possible not to pay attention to the generation of tensile stress in the analysis of boundary value problems.

\section{References}

1. M. Ohmaki, Ph.D thesis of Kyoto University, 158162 (1974) in Japanese

2. P.I. Lewin, J.B. Burland, Geotechnique, 20, 3, 259262 (1971)

3. M.A. El-Shoby, Proc. of $7^{\text {th }}$ ICSMFE, 1, 111-119 (1969)

4. T.Nakai, T. Hoshikawa, Proc. of $2^{\text {nd }}$ Chubu Symposium of Geotechnical Engineering, Chubu Branch of JGS, 125-128 (1990) in Japanese

5. A. Ishikawa, K. Terada, T. Kyoya, Y. Shamoto, Proc. of JSCE, 66,1, 145-155 (2010) in Japanese

6. T. Nakai, M. Hinokio, Soils and Foundations, 44, 2, 53-70 (2004)

7. T. Nakai, H.M. Shahin, M. Kikumoto, H. Kyokawa, F. Zhang, M.M. Farias, Soils and Foundations, 51, 6, 1149-1168 (2011)

8. T. Nakai, Constitutive Modelling of Geomaterials, (CRC press, 2012)

9. T. Nakai, Y Mihara, Soils and Foundations, 24, 2, 8294 (1984)

10. K. Hashiguchi, Journal of Applied Mechanics, ASME, 102, 2, 266-272 (1980)

\section{Appendix}

Rate type formulation of the proposed non-linear elastic model is derived referring to the method described in the paper [5].

Stress $\sigma_{i j}$ and (elastic) strain $\varepsilon_{k l}$ is expressed using their principal values and eigen vectors as follows:

$$
\sigma_{i j}=\sum_{\alpha=1}^{3} \sigma^{(\alpha)} n_{i}^{(\alpha)} n_{j}^{(\alpha)}, \quad \varepsilon_{k l}=\sum_{\alpha=1}^{3} \varepsilon^{(\alpha)} n_{k}^{(\alpha)} n_{l}^{(\alpha)}
$$

$$
\begin{aligned}
& \dot{\sigma}_{i j}=\left(\sum_{\alpha=1}^{3} \sigma^{(\alpha)} n_{i}^{(\alpha)} n_{j}^{(\alpha)}\right)^{\bullet} \\
& =\sum_{\alpha=1}^{3} \dot{\sigma}^{(\alpha)} n_{i}^{(\alpha)} n_{j}^{(\alpha)}+\sum_{\alpha=1}^{3} \sigma^{(\alpha)}\left(n_{i}^{(\alpha)} n_{j}^{(\alpha)}\right)^{\bullet} \\
& =\sum_{\alpha=1}^{3} \frac{\partial \sigma^{(\alpha)}}{\partial \varepsilon_{p q}} n_{i}^{(\alpha)} n_{j}^{(\alpha)} \dot{\varepsilon}_{p q}+\sum_{\alpha=1}^{3} \sigma^{(\alpha)}\left(n_{i}^{(\alpha)} n_{j}^{(\alpha)}\right)^{\bullet} \\
& =\sum_{\alpha=1}^{3} \frac{\partial \sigma^{(\alpha)}}{\partial \varepsilon_{p q}} n_{i}^{(\alpha)} n_{j}^{(\alpha)}\left(\sum_{\beta=1}^{3} \varepsilon^{(\beta)} n_{p}^{(\beta)} n_{q}^{(\beta)}\right)^{\bullet}+\sum_{\alpha=1}^{3} \sigma^{(\alpha)}\left(n_{i}^{(\alpha)} n_{j}^{(\alpha)}\right)^{\bullet} \\
& =\sum_{\alpha=1}^{3} \frac{\partial \sigma^{(\alpha)}}{\partial \varepsilon_{p q}} n_{i}^{(\alpha)} n_{j}^{(\alpha)}\left(\sum_{\beta=1}^{3} \dot{\varepsilon}^{(\beta)} n_{p}^{(\beta)} n_{q}^{(\beta)}+\sum_{\beta=1}^{3} \varepsilon^{(\beta)}\left(n_{p}^{(\beta)} n_{q}^{(\beta)}\right)^{\bullet}\right) \\
& +\sum_{\alpha=1}^{3} \sigma^{(\alpha)}\left(n_{i}^{(\alpha)} n_{j}^{(\alpha)}\right)^{\bullet} \\
& =\sum_{\alpha=1}^{3} \sum_{\beta=1}^{3} \frac{\partial \sigma^{(\alpha)}}{\partial \varepsilon_{p q}} n_{i}^{(\alpha)} n_{j}^{(\alpha)} n_{p}^{(\beta)} n_{q}^{(\beta)} \dot{\varepsilon}^{(\beta)} \\
& +\left(\sum_{\gamma=1}^{3} \sum_{\alpha=1}^{3} \frac{\partial \sigma^{(\gamma)}}{\partial \varepsilon_{p q}} n_{i}^{(\gamma)} n_{j}^{(\gamma)} \varepsilon^{(\alpha)}+\sum_{\alpha=1}^{3} \sigma^{(\alpha)} \delta_{p i} \delta_{q j}\right)\left(n_{p}^{(\alpha)} n_{q}^{(\alpha)}\right)^{\bullet} \\
& =\sum_{\alpha=1}^{3} \sum_{\beta=1}^{3} \frac{\partial \sigma^{(\alpha)}}{\partial \varepsilon_{p q}} n_{i}^{(\alpha)} n_{j}^{(\alpha)} n_{p}^{(\beta)} n_{q}^{(\beta)} \dot{\varepsilon}^{(\beta)} \\
& +\sum_{\substack{\alpha=1 \\
\alpha \neq=1}}^{3} \sum_{\substack{\beta \neq \beta \\
(\gamma=1}}^{3}\left(\sum_{\gamma=1}^{\partial \sigma^{(\gamma)}} n_{i}^{(\gamma)} n_{j}^{(\gamma)}+\frac{\sigma^{(\alpha)}-\sigma^{(\beta)}}{\varepsilon^{(\alpha)}-\varepsilon^{(\beta)}} \delta_{p i} \delta_{q j}\right) \\
& \text { ధ }\left(\varepsilon^{(\alpha)}-\varepsilon^{(\beta)}\right) W_{\alpha \beta} n_{p}^{(\alpha)} n_{q}^{(\beta)}
\end{aligned}
$$

Eq. (A2) is written using rate of $\varepsilon_{k l}$ as:

$$
\dot{\sigma}_{i j}=\dot{\sigma}_{i j}^{1}+\dot{\sigma}_{i j}^{2}=\left(C_{i j k l}^{1}+C_{i j k l}^{2}\right) \dot{\varepsilon}_{k l}
$$

where:

$$
\begin{aligned}
& \left(\begin{array}{l}
C_{i j k l}^{1}=\sum_{\alpha=1}^{3} \sum_{\beta=1}^{3} \frac{\partial \sigma^{(\alpha)}}{\partial \varepsilon_{p q}} n_{p}^{(\beta)} n_{q}^{(\beta)} n_{i}^{(\alpha)} n_{j}^{(\alpha)} n_{k}^{(\beta)} n_{l}^{(\beta)} \\
C_{i j k l}^{2}=\sum_{\substack{\alpha=1 \\
\beta}}^{3} \sum_{\substack{\beta=1 \\
\alpha \neq \beta}}^{3}\left(\sum_{\gamma=1}^{3} \frac{\partial \sigma^{(\gamma)}}{\partial \varepsilon_{p q}} n_{i}^{(\gamma)} n_{j}^{(\gamma)}+\frac{\sigma^{(\alpha)}-\sigma^{(\beta)}}{\varepsilon^{(\alpha)}-\varepsilon^{(\beta)}} \delta_{p i} \delta_{q j}\right)
\end{array}\right. \\
& {\left[n_{p}^{(\alpha)} n_{q}^{(\beta)} \frac{1}{2}\left(n_{k}^{(\alpha)} n_{l}^{(\beta)}+n_{k}^{(\beta)} n_{l}^{(\alpha)}\right)\right.}
\end{aligned}
$$

Also, the partial derivative of principal stress $\sigma^{(\alpha)}$ in Eq. (5) with respect to strain $\varepsilon_{p q}$ is given by:

Then, rate of $\sigma_{i j}$ is calculated as: 


$$
\begin{aligned}
& \frac{\partial \sigma^{(\alpha)}}{\partial \varepsilon_{p q}}= \frac{\partial}{\partial \varepsilon_{p q}}\left(P_{a} \exp \left\{\Delta\left(\varepsilon^{(\alpha)}+\frac{v}{1-v} \varepsilon^{(\beta)}+\frac{v}{1-v} \varepsilon^{(\gamma)}\right)\right\}\right) \\
&= \Delta\left(n_{p}^{(\alpha)} n_{q}^{(\alpha)}+\frac{v}{1-v} n_{p}^{(\beta)} n_{q}^{(\beta)}+\frac{v}{1-v} n_{p}^{(\gamma)} n_{q}^{(\gamma)}\right) \\
& \square P_{a} \exp \left\{\Delta\left(\varepsilon^{(\alpha)}+\frac{v}{1-v} \varepsilon^{(\beta)}+\frac{v}{1-v} \varepsilon^{(\gamma)}\right)\right\} \\
&= \Delta\left(n_{p}^{(\alpha)} n_{q}^{(\alpha)}+\frac{v}{1-v} n_{p}^{(\beta)} n_{q}^{(\beta)}+\frac{v}{1-v} n_{p}^{(\gamma)} n_{q}^{(\gamma)}\right) \sigma^{(\alpha)} \\
&(\alpha, \beta, \gamma=1,2,3 \& \alpha \neq \beta \neq \gamma)
\end{aligned}
$$

In Eq. (A4), when $\varepsilon^{(\alpha)}=\varepsilon^{(\beta)}\left(\sigma^{(\alpha)}=\sigma^{(\beta)}\right)$,

$$
\frac{\sigma^{(\alpha)}-\sigma^{(\beta)}}{\varepsilon^{(\alpha)}-\varepsilon^{(\beta)}} \text { in Eq. (A4) is replaced by } \frac{\partial \sigma^{(\alpha)}}{\partial \varepsilon^{(\alpha)}}
$$

(L'Hopital's rule) 